\title{
A Case of Delayed-Onset Propionibacterium acnes Endophthalmitis after Cataract Surgery with Implantation of a Preloaded Intraocular Lens
}

\author{
Yuki Hayashi Hiroshi Eguchi Tatsuro Miyamoto \\ Masayuki Inoue Yoshinori Mitamura \\ Department of Ophthalmology, Institute of Health Biosciences, The University \\ of Tokushima Graduate School, Tokushima, Japan
}

\section{Key Words}

Endophthalmitis - Preloaded intraocular lens - Propionibacterium acnes .

Scanning electron microscopy

\begin{abstract}
Purpose: To report a case of delayed-onset endophthalmitis after implantation of a preloaded intraocular lens $(\mathrm{IOL})$ and examine the surgically removed IOL by scanning electron microscopy (SEM).
\end{abstract}

Case: A 77-year-old female underwent uneventful phacoemulsification and aspiration with preloaded silicone $\mathrm{IOL}$ implantation. Since intraocular inflammation unexpectedly worsened 1 month after the surgery, she was referred to our hospital. Her visual acuity was hand motion in the left eye. Hypopyon and fibrin formation were observed in the anterior chamber. A diagnosis of postoperative delayed-onset endophthalmitis was made, and vitrectomy with anterior chamber wash-out was performed. As intraocular inflammation remained unchanged postoperatively, an additional surgery with IOL removal was performed. We cultivated the surgically removed samples of aqueous humor and vitreous fluid under both aerobic and anaerobic conditions, performed 16S rDNA clone library analysis of these clinical samples, and examined the removed IOL by SEM.

Result: Inflammation subsided after the re-operation. Although cultures of aqueous and vitreous samples were negative, DNA of Propionibacterium acnes was detected in the aqueous humor. The SEM images showed that the rod bacteria and biofilm-like material formed on the tip of the IOL haptic.

Conclusion: Delayed-onset endophthalmitis may occur after uneventful implantation of a preloaded IOL. The SEM findings suggested that the tip of the preloaded IOL haptic might scratch bacteria which adhered to the tip of the injector nozzle when the IOL was inserted 
into the anterior chamber. In some cases with delayed-onset endophthalmitis, IOL removal is needed to eliminate the bacteria which adhere to the tip of the IOL haptic.

\section{Introduction}

Infectious endophthalmitis is one of the severe complications of intraocular surgery. Delayed-onset endophthalmitis is most often induced by low-virulent bacteria, such as Propionibacterium acnes [1]. The precise mechanisms by which these bacteria cause endophthalmitis after cataract surgery remain unknown. Previous reports on lowgrade endophthalmitis after cataract surgery showed bacteria on the surface of the removed intraocular lenses (IOLs) by scanning electron microscopy (SEM) [2,3]. However, these bacteria were detected almost only on the IOL optic, and the presence of bacteria on the IOL haptic has not been examined sufficiently [4].

In recent years, the use of injectors for IOL implantation has been increasing. The advantage of using an injector rather than a forceps is a reduced risk for bacterial entry into the eye because the IOL has no direct contact with the incision or the operative fields [5]. On the contrary, bacterial contamination of the anterior chamber is not prevented completely despite the use of injectors for IOL implantation [6].

Herein, we report the case of a patient with P. acnes endophthalmitis after cataract surgery with the preloaded injectable IOL implantation, and suggest the possibility that delayed-onset endophthalmitis can occur even when preloaded IOL is implanted uneventfully. The SEM images revealed bacterial adherence to the tip of the IOL haptic.

\section{Case Report}

A 77-year-old female underwent uneventful phacoemulsification and aspiration in her left eye in another eye clinic. A preloaded IOL (3-piece silicone IOL; Nidek, Gamagori, Japan) was implanted through a scleral tunnel incision. Her past history included hypertension, diabetes mellitus, angina pectoris, and diabetic retinopathy. One day after the surgery, only mild iritis was observed in the left eye. One week after the surgery, the best-corrected visual acuity (BCVA) was 1.0, and slight inflammation was observed in the anterior chamber. Four weeks after the surgery, hypopyon was observed, leading the patient to consult us. The BCVA was 0.9 in the right eye and hand motion in the left eye. Intraocular pressure was $14 \mathrm{~mm} \mathrm{Hg}$ in the right eye and $18 \mathrm{~mm} \mathrm{Hg}$ in the left eye. Slit-lamp biomicroscopy showed many mutton-fat keratic precipitates and severe iritis with fibrin formation and hypopyon ( fig. 1). The capsular bag and ocular fundus could not be seen in detail because of the haze. The presence of white plaques on the posterior capsule or IOL, which is a characteristic finding for $P$. acnes endophthalmitis, remained uncertain. No abnormal shadow was detected in the vitreous cavity using B-mode echographic examination. Electroretinogram elicited by a bright white flash showed slightly decreased amplitude in her left eye.

According to these findings, a diagnosis of delayed-onset infectious endophthalmitis was made. On the day of initial consultation, vancomycin hydrochloride $(1.0 \mathrm{mg} / 0.1 \mathrm{ml})$ and ceftazidime hydrate $(2.0 \mathrm{mg} / 0.1 \mathrm{ml})$ were injected into the vitreous cavity because we could not perform emergency surgery for several hours. The patient underwent pars plana vitrectomy and anterior chamber lavage on the night of the same day. Intraoperatively, vitreous opacity was found after anterior chamber lavage, and the vitreous cavity and anterior chamber were irrigated with vancomycin and ceftazidime. Posterior capsulectomy was performed so that antibiotics might reach into the capsular bag. Vitrectomy and partial capsulectomy without IOL removal are considered to be one of the first 
surgical steps which can lead to resolution of endophthalmitis [1]. Thus, the IOL was not removed in the first operation.

Postoperatively, autogenously produced eye drop of vancomycin hydrochloride $1 \%$ and moxifloxacin hydrochloride eye drop (Vegemox ${ }^{\circledast}$ Ophthalmic Solution 0.5\%; Alcon, Tokyo, Japan), and systemic imipenem/cilastatin sodium (Thienam ${ }^{\circledR}$ for intravenous drip infusion $0.5 \mathrm{~g}$, MSD, Tokyo, Japan) administration were initiated. However, the fibrin content in the anterior chamber increased gradually. Four days after the first surgery, an additional vitrectomy with IOL removal was performed. Since the first surgery was not effective, we considered that existence of IOL might induce bacteria viability in the capsular bag. Thus, IOL exchange was not carried out in the second surgery. The posterior surface of the anterior lens capsule was polished to remove the inflammatory cells and fibrin. The extracted silicone IOL was fixed in $4 \%$ paraformaldehyde and processed for SEM (JSM6320F; JEOL, Tokyo, Japan).

Gram staining and Wright-Giemsa staining of aqueous and vitreous samples showed no bacteria. Aerobic and anaerobic cultures of the aqueous and vitreous samples were also negative.

For 16S rDNA sequence analysis, the bacterial DNAs in the aqueous and vitreous samples were extracted and purified using Extrap Soil DNA Kit Plus Ver. 2 (Nippon Steel Kankyo Engineering, Tokyo, Japan). The $16 \mathrm{~S}$ rDNA gene fragments were amplified with the purified DNAs as a template by use of universal primer Bac1392R (ACGGGCGGTGTGAC) [7]. After cloning those amplified products, the sequences of 48 clones were analyzed with the primer 27f (AGAGTTTGATCMTGGCTCAG) [8], and these homologies were searched in an official database. Several clones of $P$. acnes were detected from the aqueous humor by use of this $16 \mathrm{~S}$ rDNA clone library analysis.

In the SEM images, many rod shaped bacteria were observed on the tip of the IOL haptic ( fig. 2 ), which was compatible with the result of the DNA clone library analysis and the clinical course of delayed onset inflammation. Each bacterium seemed to be attached by biofilm-like material to other bacteria and the IOL haptic.

After removal of the IOL, the ocular inflammation subsided rapidly. Seven months after the IOL removal, the BCVA improved to 0.4. Fundus examination disclosed a simple stage of diabetic retinopathy. IOL re-implantation is planned.

\section{Discussion}

Delayed-onset endophthalmitis is most often induced by low-virulent anaerobic bacteria such as $P$. acnes [1]. These bacteria can grow in the capsular bag where oxygen pressure is low [9]. Bacteria were reported to be detected on the surface of the IOL optic and in the capsular bag in endophthalmitis after cataract surgery [2, 3]. In our present case, bacteria were observed on the tip of the IOL haptic by SEM.

Regarding the first surgical steps for P. acnes endophthalmitis, pars plana vitrectomy, partial capsulectomy and intraocular antibiotic injection without IOL removal are reported to be usually successful in long-term follow-up [1]. In patients with recurrent inflammation, vitrectomy with total capsular bag removal, intraocular antibiotic injection, and IOL exchange or removal should be taken into account $[1,10]$. Although we performed irrigation of antibiotics and posterior capsulectomy in the first vitrectomy, the ocular infection remained unchanged. One possible reason why the first surgical approach failed is that the quantity of antibiotics in the capsular bag failed to exhibit a bactericidal effect in the area between the tip of the IOL haptic and the equatorial part of the capsular bag. Possibly, a sufficient amount of antibiotics did not reach to the capsular bag because the capsule was sealed around the IOL. 
Another possible reason is that bacteria on the tip of the IOL haptic might be protected by a biofilm. P. acnes are known to produce a biofilm on the artificial devices. Lai et al. [11] reported that $P$. acnes DNA was detected in an explanted IOL by polymerase chain reaction in a case of chronic pseudophakic endophthalmitis. SEM findings showed a coating of biofilm-like aggregates, diphtheroid particles, and crystals on the surface of the IOL, suggesting a bacterial biofilm on the surface of the IOL [11]. In our case, biofilm-like material on the IOL haptic was observed in SEM images. It was uncertain whether the material covering the rod-shaped bacteria was real biofilm because we could not investigate the biofilm ability of this clinical strain. However, we believe that the IOL removal was essential for complete remission of the inflammation which supports our expertise. Teichmann [12] also reported $P$. acnes endophthalmitis requiring IOL removal after failure of medical therapy.

IOL implantation using an injector is advantageous for reducing the risk of bacterial entry into the eye because the IOL has no direct contact with the incision or the operative fields. Mayer et al. [5] reported that usage of injectable IOLs was associated with a lower risk of postoperative endophthalmitis $(0.028 \%)$ as compared with usage of foldable IOLs (1.21\%). Our present case suggests that endophthalmitis may occur even though a preloaded injectable IOL is used.

In the present case, the pathway of bacterial entry into the eye remains unclear. However, it is possible that the bacteria around the incision adhered to the tip of the injector nozzle when the injector was inserted into the anterior chamber. The tip of the IOL haptic might scratch the bacteria which adhered to the tip of the injector nozzle when the IOL was inserted into the anterior chamber. Shimada et al. [13] reported that repeated irrigation of the operative field with povidone-iodine at a concentration of $0.25 \%$ achieved an extremely low bacterial contamination rate in the anterior chamber at the completion of surgery in cataract surgery. To reduce the risk of bacterial entry into the eyes, wash-out of the operative fields with povidone-iodine before the IOL implantation is considered to be effective.

In conclusion, delayed-onset $P$. acnes endophthalmitis may occur after cataract surgery even if a preloaded injectable IOL is implanted uneventfully. The SEM findings suggested that the tip of the preloaded IOL haptic might scratch bacteria which adhered to the tip of injector nozzle in the present case. A sufficient irrigation of antibiotics in the capsular bag is essential during the surgery for endophthalmitis. In some cases, IOL removal is needed to eliminate the bacteria which adhere to the tip of the IOL haptic.

\section{Disclosure Statement}

The authors have no conflicts of interest to disclose. 


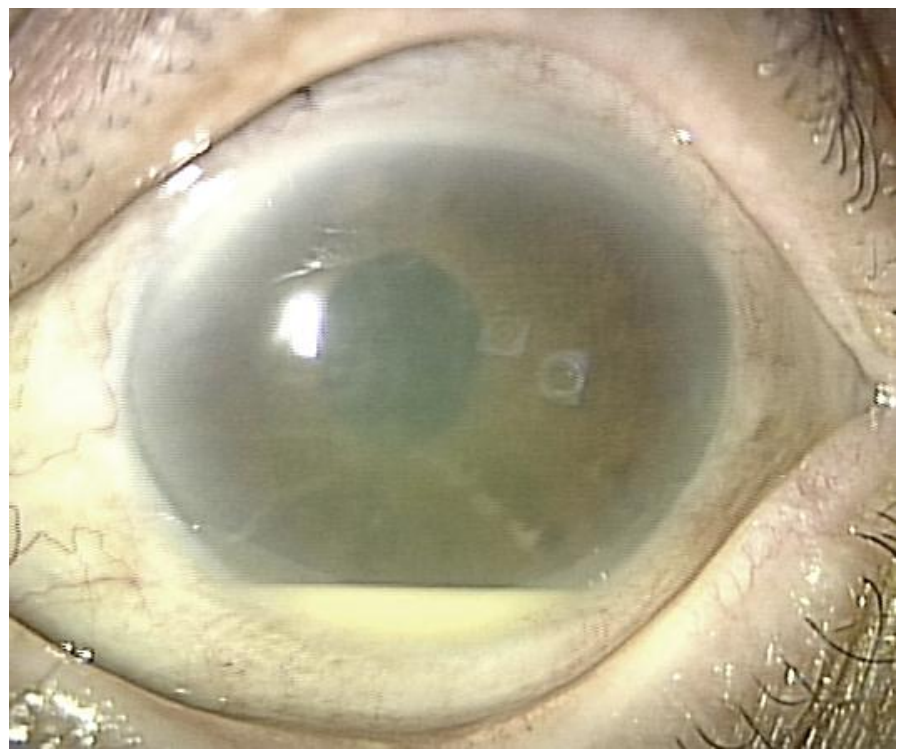

Fig. 1. Anterior segment findings 4 weeks after cataract surgery. Hypopyon and fibrin formation in the anterior chamber and many mutton-fat keratic precipitates are observed. 

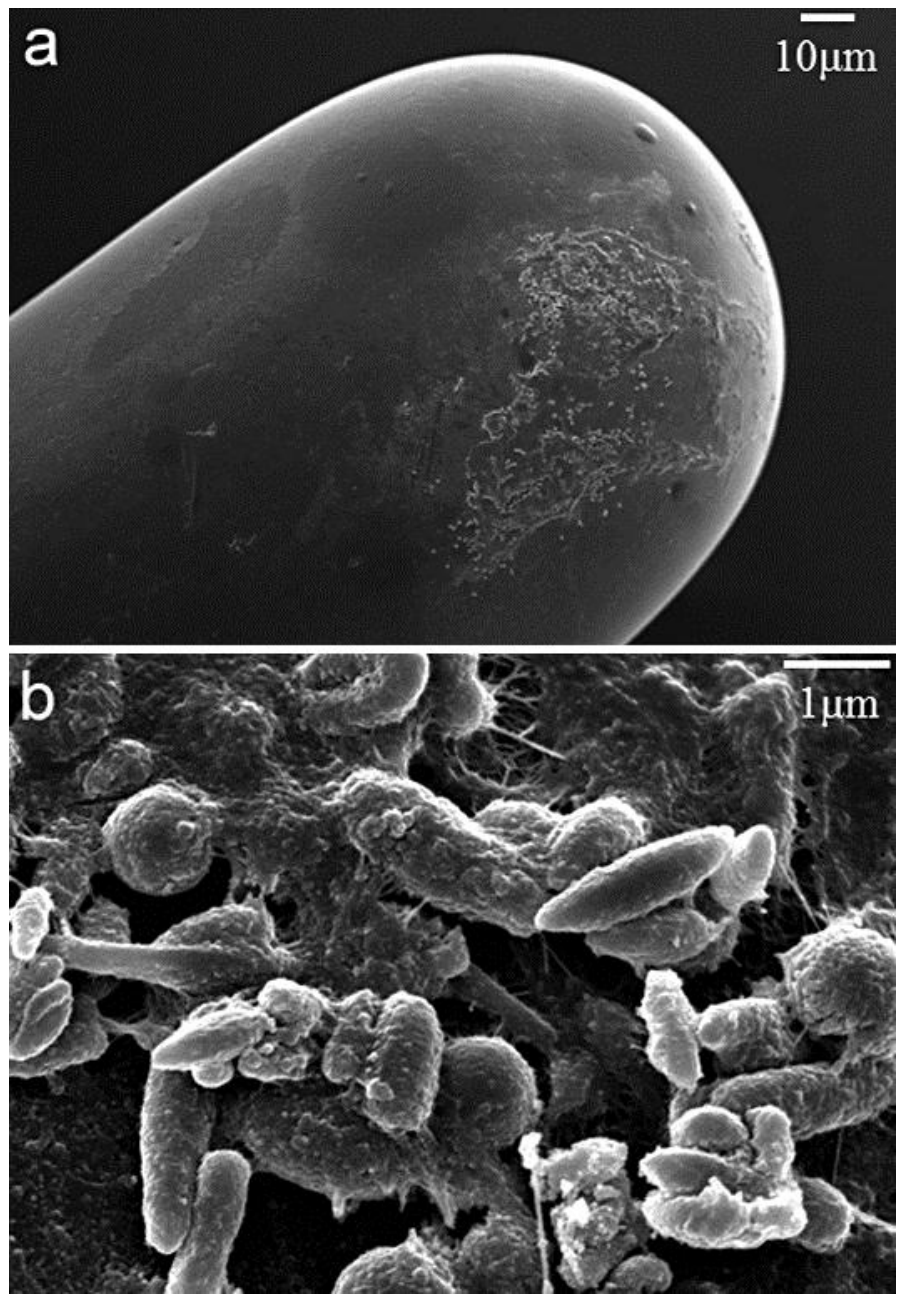

Fig. 2. Scanning electron photomicrograph of the haptic loop of the removed IOL. a Many bacteria are visible on the tip of the IOL haptic. $\mathbf{b}$ Each bacterium is attached by biofilm-like material to other bacteria and the IOL haptic.

\section{References}

1 Clark WL, Kaiser PK, Flynn HW Jr, Belfort A, Miller D, Meisler DM: Treatment strategies and visual acuity outcomes in chronic postoperative Propionibacterium acnes endophthalmitis. Ophthalmology 1999;106:1665-1670.

2 Busin M, Cusumano A, Spitznas M: Intraocular lens removal from eye with chronic low-grade endophthalmitis. J Cataract Refract Surg 1995;21:679-684.

-3 Suzuki T, Uno T, Kawamura Y, Joko T, Ohashi Y: Postoperative low-grade endophthalmitis caused by biofilm-producing coccus bacteria attached to posterior surface of intraocular lens. J Cataract Refract Surg 2005;31:2019-2020.

-4 Saika S, Kawashima Y, Miyamoto T, Okada Y, Tanaka S, Ohnishi Y, Ooshima A, Yamanaka A: Pathological findings in lens capsule and silicone intraocular lens extracted from eye with chronic infectious endophthalmitis. Jpn J Ophthalmol 1998;42:456-460.

5 Mayer E, Cadman D, Ewings P, Twomey JM, Gray RH, Claridge KG, Hakin KN, Bates AK: A 10 year retrospective survey of cataract surgery and endophthalmitis in a single eye unit: injectable lenses lower the incidence of endophthalmitis. Br J Ophthalmol 2003;87:867-869. 
6 Bausz M, Fodor E, Resch MD, Kristóf K: Bacterial contamination in the anterior chamber after povidoneiodine application and the effect of the lens implantation. J Cataract Refract Surg 2006;32:1691-1695.

-7 Marchesi JR, Sato T, Weightman AJ, Martin TA, Fry JC, Hiom SJ, Dymock D, Wade WG: Design and evaluation of useful bacterium-specific PCR primers that amplify genes coding for bacterial 16S rRNA. Appl Environ Microbiol 1998;64:795-799.

-8 Ritalahti KM, Amos BK, Sung Y, Wu Q, Koenigsberg SS, Löffler FE: Quantitative PCR targeting 16S rRNA and reductive dehalogenase genes simultaneously monitors multiple Dehalococcoides strains. Appl Environ Microbiol 2006;72:2765-2774.

-9 Ormerod LD, Edelstein MA, Schmidt GJ, Juarez RS, Finegold SM, Smith RE: The intraocular environment and experimental anaerobic bacterial endophthalmitis. Arch Ophthalmol 1987;105:1571-1575.

10 Aldave AJ, Stein JD, Deramo VA, Shah GK, Fischer DH, Maguire JI: Treatment strategies for postoperative Propionibacterium acnes endophthalmitis. Ophthalmology 1999;106:2395-2401.

11 Lai JY, Chen KH, Lin YC, Hsu WM, Lee SM: Propionibacterium acnes DNA from an explanted intraocular lens detected by polymerase chain reaction in a case of chronic pseudophakic endophthalmitis. J Cataract Refract Surg 2006;32:522-525.

-12 Teichmann KD: Propionibacterium acnes endophthalmitis requiring intraocular lens removal after failure of medical therapy. J Cataract Refract Surg 2000;26:1085-1088.

13 Shimada H, Arai S, Nakashizuka H, Hattori T, Yuzawa M: Reduction of anterior chamber contamination rate after cataract surgery by intraoperative surface irrigation with $0.25 \%$ povidone-iodine. Am J Ophthalmol 2011;151:11-17. 